\title{
Avaliação antropométrica e análise dietética de pré-escolares em centros educacionais municipais no sul de Minas Gerais
}

\author{
Anthropometric evaluation and food intake \\ of preschool children at municipal educational centers, \\ in South of M inas Gerais State, Brazil
}

Hércia Stampini Duarte $M$ artino ${ }^{1}$

Andreza Campos Ferreira ${ }^{2}$

Cristina N ovack Amaral Pereira ${ }^{2}$

Roberta Ribeiro Silva²

${ }^{1}$ Departamento de N utrição eSaúde, Universidade Federal deViçosa. Campus Universitário $\mathrm{s} / \mathrm{n}$. 36570-000 Viçosa M G. hercia@ufv.br

${ }^{2}$ Departamento deN utrição, UniversidadeFederal de Alfenas.
Abstract The objective of this article is to evaluate socioeconomic conditions, nutritional status and food intake of preschool children assisted at Municipal Educational Centers (CEM s) of Alfenas, $M$ inas Gerais State, Brazil. A transversestudy involving 186 children was performed. The socioeconomic profile, weight, height, age, sex and food intake by a direct weighting tool were evaluated. It was observed an income family of one to three minimum wages, incomplete fundamental learning, private house with basic sanitation, four to fiveinhabitants per house of whom two worked at most. Theaverage agewas $49.2 \pm 12.9$ months. The stunting rates were more prevalent among the children $(20.5 \%)$. The whole, $72.6 \%$ of children have energy intake below Estimated Energy Intake (EER). H owever, when stratifying for age group, $78.4 \%$ of the children from 1 to 3 years old have high energy intake and $74.1 \%$ from 4 to 6 years old have inadequate energy intake. All children presented consumption below the Adequate Intake (AI) of calcium and most of them below to Estimated Average Requirement (EAR) of iron. Despite some biosocioeconomic factors be favorable to the nutritional status, malnutrition, and inadequate nutrient intake were present, which demanded theimplementation of nutritional programs in CEM s.

Key words Nutritional status, Food intake, Nutrients, Preschool children, WHO child growth standards
Resumo O bjetivou-se avaliar as condições socioeconômicas, o estado nutricional e o consumo alimentar de préescolares assistidos pelos Centros EducacionaisM unicipais (CEM) deAlfenas (M G). Foi realizado um estudo transversal, com 186 crianças. Avaliou-se o perfil socioeconômico, peso, estatura, idade, sexo e o consumo alimentar pelo instrumento de pesagem di reta dosalimentos. $\mathrm{Ob}$ servou-se renda familiar deum a três salários mínimos, ensino fundamental incompleto, casa própria com saneamento básico, quatro a cinco habitantes por domićlio, dos quais, na maioria dois trabalhavam. A média de idade foi de 49,2 $\pm 12,9$ meses. A baixa estatura foi 0 desvio nutricional mais prevalente $(20,5 \%) .72,6 \%$ das criançasapresentaram ingestão energética abai xo da necessidade energética estimada (EER). Ao estratificar por faixa etária, deum a trêsanos, $78,4 \%$ das crianças apresentaram ingestão acima da EER e de quatro a seis anos, 74,1\% apresentaram ingestão abaixo da EER. Todas as crianças apresentaram baixo consumo de cálcio e a maioria apresentou consumo abaixo da necessidade média estimada (EAR) para ferro. A pesar de alguns fatores biossocioeconômicos serem favoráveis, os desvios nutricionais e a ingestão inadequada de nutrientes estavam presentes, tornando-se necessária implementação de programa de controle nutricional nos CEM .

Palavras-chave Estado nutricional, Consumo alimentar, Nutrientes, Préescolar, Padrão decrescimento da WHO 
Introdução

As deficiências de nutrientes são fatores de risco à saúde e sobrevivência de grupos vulneráveis, em especial os pré-escolares, sendo, em geral, causadas pela ingestão dietética insuficiente ${ }^{1}$. No Brasil, estudos em creches identificaram associação positiva entre déficit decrescimento e desenvolvimento e consumo alimentar inadequado ${ }^{2}$; a presença de sobrepeso eobesidadeem detrimento à desnutrição protéico-energética ${ }^{3-5} \mathrm{e}$ a baixa estatura e o excesso de peso $0^{6,7}$.

$\mathrm{Na}$ tentativa de combater os agravos nutricionais nas crianças brasileiras, o mais antigo programa social do governo federal, o Programa Nacional de Alimentação Escolar (PNAE), na área de alimentação e nutrição, vem buscando promover a segurança alimentar enutricional por meio da formação de hábitos alimentares saudáveis, promoção do crescimento e desenvolvimento das crianças e melhoria da apren dizagem erendimento escolar. Asúltimas estatísticas mostraram a cobertura de 36,3 milhões de crianças e jovens, $21 \%$ da população brasileira ${ }^{8}$.

Segundo o Fundo Nacional de Desenvolvimento da Educação (FNDE), as crianças em creches, em período integral, devem receber os cardápios com refeições bal anceadas que venham a suprir no mínimo $70 \%$ das necessidades nutricionais diárias 8 . 0 diagnóstico eacompanhamento dasituação nutricional de crianças institucionalizadas tornam-se importante, para que os Centros Educacionais Municipais (CEM), anteriormente denominados creches municipais, possam propor estratégias para atender as diretrizes da FNDE, promovendo o desenvolvimento integral dacriança. Diantedeste contexto, o objetivo deste trabalho foi avaliar as condições socioeconômicas, o estado nutricional e o consumo alimentar em pré-escolares assistidos pelos Centros Educacionais M unicipais de Alfenas (MG).

\section{Métodos}

População estudada

Foi realizado um estudo transversal, descritivo, deagosto de 2004 a julho de 2005, em todos os quatros Centros Educacionais Municipais (CEM) de Alfenas (M G). Da população de 236 crianças, foi calculada uma amostra de 189 no estágio de vida entre 16 a 82 meses, de ambos os sexos, com prevalência entre 10 a $15 \%$ e erros de precisão de 5 a $10 \%$, utilizando para o cálculo 0 programa Epi Info 6 versão 6.04 . 0 processo de seleção destas crianças foi realizado conforme amostragem aleatória simples. Da amostra calculada, oito crianças abandonaram a pesquisa.

Os CEM em que o trabalho foi conduzido funcionavam em regime de oito horas, fornecendo, nesse período, entre quatro e cinco refeições: desjejum, colação, almoço, lanche da tarde 1 e lanche da tarde 2 , de segunda a sexta-feira. Foram realizadas reuniões com a finalidade de informar e esclarecer professores, pais ou responsáveis, sobre os objetivos do trabalho e procedimentos realizados.

\section{Coleta dos dados}

O trabalho de coleta de dados foi iniciado após aprovação do Comitê de Ética em Pesquisa Humana da Universidade Federal de Alfenas, do consentimento da Secretaria M unicipal de Educação e da assinatura do termo de consentimento informado pelos pais das crianças, sendo que a estes foi garantido 0 anonimato e 0 direito de deixar a pesquisa em qualquer fase de execução.

Os dados socioeconômicos foram obtidos a partir de um questionário preenchido pelos pais ou responsáveis pelas crianças. As variáveis analisadas foram renda familiar, escolaridade dos pais, condições de moradia, número de pessoas por domicílio e, destas, quais contribuíam com a renda familiar.

\section{Avaliação antropométrica}

Foram adotadas as técnicas de Jelliffe ${ }^{9}$ para a obtenção do peso e comprimento/estatura. Para a medida do peso, foi utilizada balança portátil, digital, com capacidade de 150 quilos e sensibilidade de 50 gramas. As medidas de comprimento foram feitas por meio de antropômetro e infantômetro com extensão de dois metros, dividida em $\mathrm{cm}$ e subdividida em milímetros.

A determinação dosíndices peso/idade(P/I), peso/estatura (P/E) e estatura/idade (E/I) foram feitos pelo critério escore-z, considerando-se o novo padrão de um estudo multicêntrico, WHO multicentre Growth Reference Study ${ }^{10}$, para avaliar o crescimento e desenvolvimento de crianças ao nascer aos cinco anos de idade. A desnutrição foi indicada pelo escore $z \leq-2$ para osíndices peso/ idade ou peso/estatura e a baixa estatura para índice $E / I \leq-2$ escore $z$ e entre -2 e -1 escore $z$ risco para baixa estatura; o risco nutricional para desnutrição foi indicado pelo escore-z $>-2 e \leq-1$. 0 indicador de sobrepeso foi o escore $z \geq+2$ 
para peso/idade ou peso/estatura; o indicador derisco nutricional para sobrepeso foi o interva$10 \geq+1$ e $<+2$ e baixa estatura $<-2$ escore $z$.

Inquérito alimentar

O consumo das refeições pelas crianças nos CEM foi avaliado pelo instrumento de pesagem direta dos alimentos. Para esse procedimento, as crianças selecionadas aleatoriamente em cada CEM foram identificadas com uma fita no braço, assim como todos os utensílios utilizados foram diferenciados nas refeições do dia.

Acompanhou-se a elaboração das refeições. A pós a finalização das preparações, foi realizada a pesagem. Antes da distribuição das refeições às crianças, a porção da preparação a ser servida foi pesada três vezes consecutivas para obter uma medida padrão, pesando-se também a refeição oferecida. A pós o consumo final das refeições, os utensílios foram recolhi dos e o resto ingestão foi pesado. As pesagens foram feitas em balança digital com capacidade de dois quilos esensibilidade de um grama.

Para a avaliação da ingestão de energia, foi utilizada a necessidade estimada de energia (Estimated Energy Requirement - EER). Para calcular a EER, foram utilizadas as equações para predição de gasto energético total nos diferentes estágios de vida, considerando idade, sexo, peso, estatura e atividade física, adotando-se como coeficientes deatividadefísica os valores de 1,13 para o sexo masculino e de 1,16 para o sexo feminino, representando um nível de atividadefísicaleve. A adequação da ingestão de energia e de macronutrientes (carboidratos, proteínas e lipídios) foi avaliada em relação às recomendações da Dietary Reference Intakes (DRI ${ }^{11}$, para os estágios de vida de um a três anos e de quatro a oito anos, utilizando a faixa de distribuição aceitável de macronutrientes. Para análise de ferro, a referência utilizada para avaliar a adequação foi a Estimated Average Requirement (EAR), utilizada para avaliar eplanejar a ingestão dietética degrupos populacionais e de cálcio, a Adequate Intake (AI), baseada em médias de ingestão observadas de um dado nutriente para determinada população ou grupo de indivíduos saudáveis ${ }^{11}$.

O consumo de nutrientes foi calculado com 0 auxílio do software Dietpro versão $4.0^{12}$, tendo como informação a tabela de composição dos alimentos U nited States Department of Agriculture ea Tabela Brasileira de Composição deAlimentos. Considerando que o consumo de nutrientes dificilmente apresenta distribuição normal, todos os nutrientes foram calculados em valores medianos.

\section{Análises estatísticas}

As análises estatísticas foram realizadas nos programas Statistical Package for the Social Sciencefor W indows(SPSS), versão 10.0, ano $2000^{13}$.

\section{Resultados}

Do total de pré-escolares, $54,3 \%$ eram do sexo masculino e $45,7 \%$, do feminino. A média deidade foi de $49,2 \pm 12,9$ meses.

Constatou-se que a maioria das famílias tinha renda entre um e três salários mínimos $(67,7 \%)$. Em relação à escolaridade, a maior parte dos pais possuía ensino fundamental incompleto. Verificou-se maior número de mães, em relação aos pais, com o ensino médio completo (Tabela 1).

A maioria das famílias possuía casa própria (74,2\%), em queresidiam de quatro a cinco membros $(57,6 \%)$. Quanto às condições sanitárias das residências, verificou-se que $100 \%$ delas dispunham de água tratada, rede de esgoto e coleta regular de lixo.

As crianças apresentaram-se eutróficas com percentuais de $58,3 \%$ para o índice P/I, 68,2 \% para P/E e 43,7\% para E/I. Observou-se desnutrição crônica ou pregressa caracterizada pela baixa estatura em $20,5 \%$ das crianças e desnutrição aguda, por baixo peso, em $4 \%$ destas. As prevalências das crianças em risco para baixo peso foram de $22,5 \%$ e $4,6 \%$ para os índices $P / I$ e $P / E$, respectivamente. 0 risco para sobrepeso prevaleceu em $13,3 \%$ para P/I e 21,9\% para P/E. As prevalências de sobrepeso entre as crianças foram de 1,9\% para P/I e 4\% para P/E (Tabela 2).

Das crianças estudadas, 72,6\% apresentaram ingestão energética abaixo da necessidade energética estimada (EER). Ao estratificar por faixa etária, entre um e três anos, $78,4 \%$ das crianças apresentaram ingestão acima da EER e, entre quatro a seis anos, 74,1\% apresentaram ingestão abaixo daEER (Figura 1).

A distribuição percentual dosmacronutrientes ficou dentro da faixa de distribuição aceitável. Para as crianças de um a três anos, o consumo mediano de proteína foi de $12,6 \%$ (35g), lipídio $30,3 \%(37,4 \mathrm{~g})$ e carboidrato $58,6 \%$ (162,4g). No ciclo de vida de quatro a seis anos, foi de 12,3 , 31,5 e $55,5 \%$, (40,8, 45,9, 182,7g) para proteína, lipídio ecarboidrato, respectivamente (Tabela3). 
Tabela 1. Caracterização dos domicílios e das famílias das crianças dos Centros Educacionais M unicipais de Alfenas (MG).

\begin{tabular}{|c|c|c|}
\hline Variáveis & $\mathrm{n}$ & $\begin{array}{c}\text { Frequência } \\
(\%)\end{array}$ \\
\hline \multicolumn{3}{|l|}{ Renda familiar } \\
\hline 0 a 1 SM & 22 & 11,8 \\
\hline 1 a 3 SM & 126 & 67,7 \\
\hline 4 a 6 SM & 35 & 18,8 \\
\hline 7 a $10 \mathrm{SM}$ & 3 & 1,6 \\
\hline \multicolumn{3}{|l|}{ Escolaridade da mãe } \\
\hline Não sabe ler & 4 & 2,2 \\
\hline Ensino fundamental incompleto & 84 & 45,2 \\
\hline Ensino fundamental completo & 70 & 37,6 \\
\hline Ensino médio incompleto & 15 & 8,1 \\
\hline Ensino médio completo & 12 & 6,5 \\
\hline Ensino superior completo & 1 & 0,5 \\
\hline \multicolumn{3}{|l|}{ Escolaridade do pai } \\
\hline Não sabe ler & 16 & 8,6 \\
\hline Ensino fundamental incompleto & 93 & 50,5 \\
\hline Ensino fundamental completo & 61 & 32,8 \\
\hline Ensino médio incompleto & 8 & 4,3 \\
\hline Ensino médio completo & 5 & 2,7 \\
\hline Ensino superior incompleto & 1 & 0,5 \\
\hline Ensino superior completo & 2 & 1,1 \\
\hline \multicolumn{3}{|l|}{ Condições de moradia } \\
\hline Casa própria & 138 & 74,2 \\
\hline Casa alugada & 48 & 25,8 \\
\hline \multicolumn{3}{|l|}{ Número de pessoas por domicílio } \\
\hline 1 a 3 & 30 & 16,1 \\
\hline 4 a 5 & 107 & 57,6 \\
\hline 6 a 9 & 49 & 26,3 \\
\hline \multicolumn{3}{|c|}{ Número de pessoas que trabalham por domicílio } \\
\hline 0 a 1 & 63 & 33,9 \\
\hline 2 a 3 & 117 & 10,9 \\
\hline 3 a 6 & 16 & 8,7 \\
\hline \multicolumn{3}{|l|}{ Água tratada } \\
\hline Sim & 186 & 100 \\
\hline Não & 0 & 0 \\
\hline \multicolumn{3}{|l|}{ Serviço de esgoto } \\
\hline $\mathrm{Sim}$ & 186 & 100 \\
\hline Não & 0 & 0 \\
\hline \multicolumn{3}{|l|}{ Lixo } \\
\hline Sim & 186 & 100 \\
\hline Não & 0 & 0 \\
\hline
\end{tabular}

A ingestão de cálcio em $100 \%$ das crianças foi inferior à Al. 0 consumo de ferro da maioria das criançasficou abaixo da EAR, 78,7\% deum a três anos e $82 \%$ de quatro a seis anos.

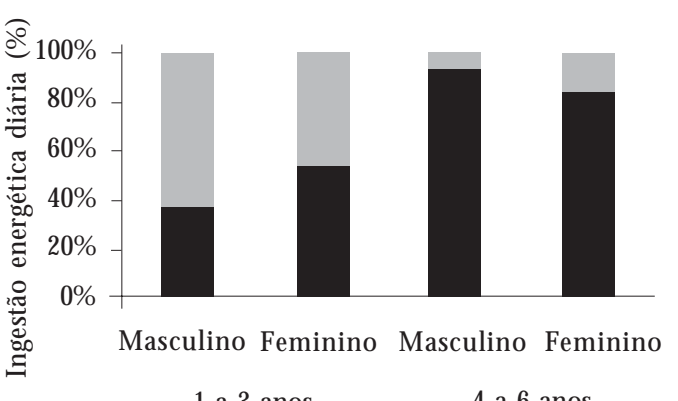

Tabela 2. Estado nutricional pelos índices $\mathrm{P} / \mathrm{I}, \mathrm{P} / \mathrm{E}$ e E/I das crianças de 0 a 5 anos dos Centros Educacionais M unicipais de Alfenas (MG).

Figura 1. Estimativa da ingestão de calorias pelas crianças dos Centros Educacionais M unicipais de Alfenas (MG).

\begin{tabular}{lrrr}
\hline $\begin{array}{l}\text { Intervalos } \\
\text { de } \boldsymbol{\chi} \text {-score }\end{array}$ & $\begin{array}{c}\mathrm{P} / \mathrm{E}(\%) \\
\mathrm{N}(\%)\end{array}$ & \multicolumn{1}{c}{$\mathrm{P} / \mathrm{N}(\%)$} \\
\hline$<-2 z$ & $31(20,5)$ & $6(4,0)$ & $2(1,3)$ \\
$-2 \mathrm{a}-1 z$ & $40(26,5)$ & $34(22,5)$ & $7(4,6)$ \\
$-1 \mathrm{a}+1 z$ & $66(43,7)$ & $88(58,3)$ & $103(68,2)$ \\
$+1 \mathrm{a}+2 z$ & $10(6,6)$ & $20(13,3)$ & $33(21,8)$ \\
$>+2 z$ & $4(2,6)$ & $3(1,9)$ & $6(4,0)$ \\
Total & $151(100)$ & $151(100)$ & $151(100)$ \\
\hline
\end{tabular}

\section{Discussão}

A maioria das famílias tinha renda entreum etrês salários mínimos. Esses resultados corroboram com os de Zöllner eFisberg ${ }^{7}$ em que a renda média das famílias foi de 3,7 salários mínimos, sendo que $34,5 \%$ das crianças pertenciam a famílias com renda inferior a 0,5 salário mínimo. No presente estudo, $11,8 \%$ das famílias tinham renda inferior a um salário mínimo. A renda per capita média das famílias estudadas foi de $1 / 3$ do salário mínimo, como nos achados de Fisberg et al. ${ }^{4}$. Os dados evidenciaram os limites no acesso à aquisição de alimentos e na utilização de outros serviços que garantam a saúde do indivíduo. Deacordo com o Instituto Brasileiro de Geografia eEsta- 
Tabela 3. Estimativas das calorias totais e de nutrientes consumidos pelas crianças dos Centros Educacionais M unicipais de Alfenas (MG).

\begin{tabular}{lcc}
\hline \multirow{2}{*}{ Nutrientes } & \multicolumn{2}{c}{ Estágio de vida } \\
\cline { 2 - 3 } & $\begin{array}{c}1 \text { a } 3 \text { anos }(n=30) \\
\text { Mediana }\left(P_{25} / P_{75}\right)\end{array}$ & 4 a 6 anos $(n=151)$ \\
\hline Calorias (Kcal) & $1108,1(811,6 / 1318,4)$ & $1305,1(1036,9 / 1599,9)$ \\
Proteína (g) & $35,0(26,4 / 44,6)$ & $40,8(32,5 / 49,8)$ \\
Lipídio (g) & $37,4(29,1 / 46,7)$ & $45,9(34,8 / 54,3)$ \\
Carboidrato (g) & $162,4(127,2 / 191,8)$ & $182,7(145,3 / 228,6)$ \\
Cálcio (mg) & $139,6(92,0 / 179,3)$ & $182,4(132,9 / 229,0)$ \\
Ferro (mg) & $4,5(3,4 / 6,2)$ & $6,0(4,3 / 7,2)$ \\
\hline P= percentil & &
\end{tabular}

$\mathrm{P}=$ percentil drão da WHO. Pelo padrão NCHS, a prevalência seria maior, 7,6\% (dado não apresentado). Tal fato ocorreu em virtude da curva do novo padrão apresentar crianças de 6 a 32 meses, com peso abaixo da mediana em relação ao $\mathrm{NCH} \mathrm{S}^{20}$.

Em assentamento de reforma agrária em $M$ inas Gerais, observaram-se prevalências consideráveis de desnutrição para crianças de 12 a 23 meses, segundo P/I (22,2\%) e E/I (33,3\%). Também foram altas e relevantes as prevalências de risco para desnutrição segundo (E/I e P/I) em mais de $30 \%$ das crianças de 24 a 60 mesese $31,1 \%$ para P/E entre crianças de 24 a 35 meses $^{21}$. Em creches de Viçosa (M G), 27,6, 19,5 e 14,9\% das crianças encontravam-se em risco para desnutrição, pelosíndices $P / I, P / E$ eE/I, respectivamente $^{17}$. Provavelmente, se o padrão de referência utilizado no estudo de Castro et al. ${ }^{17}$ fosse 0 da WHO de estudos multicêntricos ${ }^{10}$, a prevalência de baixa estatura poderia ser um pouco maior e a dedesnutrição aguda, menor, aproximando aos resultados encontrados no presente estudo.

Cavalcante et al. ${ }^{2}$ encontraram, pelo índice peso/estatura, $13,8 \%$ de desnutrição, $21,3 \% \mathrm{em}$ risco nutricional, 5,7\% em risco de sobrepeso e $1,7 \%$ apresentaram sobrepeso pelas curvas do CDC ${ }^{19}$. A prevalência de risco de sobrepeso (13,3 e21,9\%) esobrepeso (1,9 e 4\%) para osíndices P/ I e P/E, respectivamente, foi superior ao estudo supracitado. Onis et al. ${ }^{20}$, ao comparar os padrões de crescimento WHO 2007 e CDC 2000, pelo índice $P / E$, considerou que a prevalência de sobrepeso pode aumentar. Entretanto, independente do padrão adotado, as prevalências de risco para sobrepeso encontrado evidenciaram que o risco para o excesso de peso pode se tornar um problema de saúde. Em diferentes graus, essas crianças estariam vivenciando a transição nutricional: progressiva redução de déficits nutricionais e o aumento da prevalência de sobrepeso. 0 aumento do risco de obesidade quando comparada à desnutrição está relacionada com mudanças demográficas, socioeconômicas e epidemiológicas ${ }^{3}$. Assim, éfundamental o monitoramento correto e eficientepelos gestoresmunicipais, edos CEM, professores, pais, universidades e organizações não governamentais para que se possa garantir bom estado nutricional dessas crianças.

Os dados antropométricos foram comparados com o novo padrão de crescimento WHO, representativos da população mundial e com credibilidadejunto à comunidadecientífica. Entretanto, existem ainda poucos trabal hos que o utiliza.

0 método de pesagem direta, quando realizado adequadamente, é considerado o mais exa- 
to para determinar a ingestão de alimentos, podendo ser usado para crianças com resultados mais precisos que outros métodos frequentemente utilizados ${ }^{22,23}$.

Das crianças estudadas de um a três anos de idade, a maioria estava com ingestão energética recomendada ou até acima dos val ores recomendados. Porém, para a maioria das crianças de quatro a seis anos de idade, essa ingestão encontrava-se inadequada ao recomendado. No estudo de Castro et al. ${ }^{17}, 75,7 \%$ das crianças de dois a cinco anos apresentaram ingestão energética abaixo do recomendado. Em crianças na fase pré escolar, o crescimento e o desenvolvimento são intensos, a inapetência é muito comum e, quando associados à oferta inadequada de energia, poderiam explicar essa inadequação.

De modo geral, o consumo mediano de calorias fornecido pelos CEM cobre mais que 70\% das necessidades das crianças pesquisadas. Existe uma proposta que ainda não vigora, mas que recomenda que, em creches com atendimento integral, a cobertura de calorias seja pelo menos $70 \%$ das necessidades diárias ${ }^{8}$. M esmo assim, 0 aumento do consumo de calorias para crianças maiores deve ser efetivado e acompanhado com mais critério.

Quanto à distribuição percentual de calorias, o consumo alimentar das crianças estava deacordo com o preconizado para carboidratos, proteínas e lipídios ${ }^{11}$. 0 mesmo foi observado por Cruz et al . ${ }^{22}$ e Tuma et al. ${ }^{23} \mathrm{em}$ crianças assi stidas por creches municipais. Esse equilíbrio entre esses macronutrientes pode ter ocorrido, provavelmente, pela ampla faixa de distribuição percentual recomendada.

Quanto ao mineral cálcio, a ingestão foi abaixo do recomendado. 0 leiteeseus derivados, uma das principais fontes de cálcio, eram oferecidos apenas uma vez ao dia em 50\% dos CEM (dados não apresentados). Resultados semelhantes foram observados em estudo realizado em creches municipais deViçosa (M G), onde $92,8 \%$ dos préescolares apresentavam ingestão de cál cio abaixo da $\mathrm{Al}^{17}$. 0 cál cio apresentou a mediana de consumo abaixo da Al apenas na faixa etária acima de quatro a cinco anos de idade ${ }^{23}$. No presente estudo, não foi avaliado o consumo de alimentos das criançasno domicílio. M aséimportanteconsiderar que poderá haver uma maior oferta desse mineral, caso a criança receba uma refeição láctea no lar. De acordo com estudo realizado por Holland ${ }^{24}$, em creches do município de São Paulo, da mesma região, as mães costumam ofe recer pelo menos uma refeição no seu domicílio, o que colaboraria para atingir as necessidades nutricionais diárias de cálcio das crianças maiores de doze meses.

Considerando o consumo de ferro, pode-se dizer que os resultados não são satisfatórios, indicando consumo insuficiente de alimentos fonte de ferro. Esse achado corrobora com o de Cavalcante ${ }^{2}$ em crianças atendidas em serviços públicos. Ferreira et al. ${ }^{25}$ verificaram em crianças de zero a cinco anos que, à medida que aumentava a idade das crianças, diminuía a mediana de ingestão do ferro. É importante lembrar que sua quantidadenos alimentos não refletenecessariamentesua absorção pelo intestino etal fator pode agravar essa inadequação e, também, deve ser considerado para a adequada interpretação da qualidade das fontes alimentares de ferro ${ }^{26}$. Nos cardápios oferecidos pelos CEM, a maior fonte de ferro era ferro não heme, de baixa biodisponibilidade (dados não apresentados). Segundo Oliveira et al. ${ }^{27}$, em crianças com idade $\geq 24 \mathrm{me}$ ses, a densidade de ferro não heme nas refeições foi uma das variáveis explicativas para a ocorrência de anemia. Assim, tanto os CEM de Alfenas (MG) quanto as refeições no lar devem meIhorar a oferta de alimentos ricos em ferro biodisponível para corrigir ou prevenir anemias tão comuns nessa faixa etária.

\section{Conclusão}

A pesar das condições favoráveis de saneamento, habitação, escolaridade materna e socioeconômica da maioria das famílias das crianças estudadas, ficou evidenciada entre as crianças uma prevalência significante de baixa estatura. As calorias estavam adequadas para a maioria das crianças menores e inadequada para a maioria das crianças maiores. A ingestão de cálcio e ferro para a maioria das crianças apresentou-se abaixo das necessidades para todas as crianças.

Tornam-se necessários inquéritos dietéticose antropométricos mais frequentese complementares para melhorar o planejamento e a oferta da ingestão dealimentosfontes em nutrientesimportantes nesse estágio de vida. E assim atender, de forma contextualizada, as reais necessidades nutricionais das crianças estudadas por parte destas instituições. Os gestores responsáveis poderão implantar e implementar programas de alimentação específicos nos CEM, destinados às crianças, com adesão e apoio dos pais e funcionários. 


\section{Colaboradores}

HSD M artino realizou todas as etapas executadas no projeto, realizou análises estatísticas, auxiliou na interpretação dos dados, redação intelectual efinal do manuscrito; AC Ferreira executou as etapas de levantamento dos dados e participou da redação inicial do manuscrito; CN A Pereira executou as etapas de levantamento dos dados e participou da redação inicial do manuscrito; RR Silva coordenou e supervisionou todas as etapas executadas no projeto, orientou a parte metodológica e intelectual do trabalho, e auxiliou na redação do manuscrito.

\section{Referências}

1. Martins M C, Santos LM P, Santos SM C, Araújo M PN, Lima AM P, Santana LAA. Avaliação de políticas públicas de segurança alimentar e combate à fome no período 1995-2002. 3 - o Programa Nacional de Controle da Deficiência de Vitamina A. Cad Saude Publica 2007; 23(9):2081-2093.

2. Cavalcante AAM, Tinoco ALA, Cotta, RMM, Ribeiro RCL, Pereira CAS, Franceschini SCC. Consumo alimentar e estado nutricional de crianças atendidas em serviços públicos de saúde do município de Viçosa, Minas Gerais. Rev. Nutr. 2006; 19(3):321-330.

3. Batista, Filho M, Rissin A. A transição nutricional no Brasil: tendências regionais e temporais. Cad Saude Publica 2003; 19 (Sup.1): S181-191.

4. Fisberg RM, Marchioni DML, Cardoso MRA. Estado nutricional e fatores associados ao déficit de crescimento de crianças frequentadoras de creches públicas do Município de São Paulo, Brasil. Cad Saude Publica 2004; 20(3):812-817.

5. Fernandes IT, Gallo PR, Advíncula AO. Avaliação antropométrica de pré-escolares do município de Mogi-Guaçú, São Paulo: subsídio para políticas públicas de saúde. Rev. Bras. Saude Mater. Infant. 2006; 6(2):217-222.

6. Tuma RCFB, Costa THM, Schmitz BAS. Avaliação antropométrica e dietética de pré-escolares em três creches de Brasília, Distrito Federal. Rev. Bras. Saude M ater. Infant. 2005; 5(4):419-428.

7. Zöllner CC, Fisberg RM. Estado nutricional e sua relação com fatores biológicos, sociais e demográficos de crianças assistidas em creches da Prefeitura do Município de São Paulo. Rev. Bras. Saude $M$ ater. Infant. 2006; 6(3):319-328.

8. Fundo Nacional de Desenvolvimento da Educação. Alimentação Escolar. [site na Internet]. [acessado 2008 jul]. Disponível em: http://www.fnde.gov.br/ home/index.jsp?arquivo=alimentacao_escolar.html

9. Jelliffe DB. Evaluación del estado de nutrición de la comunidad con especial referencia a las encuestas en las regiones in desarrollo. Genebra: Organización Mundial de la Salud; 1968.

10. WHO Anthro 2005. Beta version Feb 17th, 2006. Software for assessing growth and development of the world's children. Geneva: WH O; 2006.

11. Institute of M edicine. Dietary Reference Intakes: The Essential Guide to Nutrient Requirements. Washington, D.C.: National Academy Press; 2006.

12. M onteiro JBR, Esteves EA. Sistema de suporte a avaliação nutricional e prescrição de dietas. Versão 4.0. Viçosa: Agromídia Software; 2005.

13. Statistical Package for the Social Science for Windows. SPSS/PC version 10.0 [programa de computador]. Chicago: SPSS; 2000.

14. Instituto Brasileiro de Geografia e Estatística. Pesquisa de orçamentos familiares, 2002-2003. Aquisição alimentar domiciliar per capita, Brasil e grandes regiões. Rio de Janeiro: IBGE; 2004.

15. Engstrom EM, Anjos LA. Déficit estatural nas crianças brasileiras: relação com condições sócio-ambientais e estado nutricional materno. Cad Saude Publica 1999;15(3):559-567. 
16. Silva M V, O metto AMH, Furtuoso COM, Pipitone $M A P$, Sturion $G L$. Acesso à creche e estado nutricional das crianças brasileiras: diferenças regionais, por faixa etária e classes de renda. Rev. Nutr. 2000; 13(3):193-199.

17. Castro TC, Novaes JF, Silva M R, Costa NM B, Franceschini SCC; Tinoco ALA, Leal PFG. Caracterização do consumo alimentar, ambiente socioeconômico e estado nutricional de pré-escolares de creches municipais. Rev. Nutr. 2005; 18(3):321-330.

18. Orellana JDY, Coimbra Jr. CEA, Lourenço AEPL, Santos RV. Nutritional status and anemia in suruí Indian children, Brazilian Amazon. J. Pediatr. 2006; 82(5):383-388.

19. Kuczmarski RJ, Ogden CL, Guo SS, Grummer-Strawn $L M$, Flegal KM, Mei $Z$, Wei $R$, Curtin LR, Roche $A F$, Johnson CL. CDC Growth Charts for the United States. Methods Development. Vital Health Stat 2000; 11(246):1-190.

20. Onis M, Garza C, Onyango AW, Borghi E. Comparison of the WHO child Growth Standard and the CDC 2000 Growth charts. J. N utr. 2007; 137:144-148.

21. Campos M TFS, Franceschini SCC, Rangel AA. Saúde e nutrição de crianças de 0 a 60 meses de um assentamento de reforma agrária, Vale do Rio Doce, MG, Brasil. Rev. Nutr. 2004; 17(2):167-176.

22. Cruz GF, Santos RS, Carvalho CM RG, Moita GC. Avaliação dietética em creches municipais de Teresina, Piauí, Brasil. Rev. Nutr. 2001; 14(1):21-32.

23. Tuma RCFB, Costa THM, Schmitz, BAS. Avaliação antropométrica e dietética de pré-escolares em três creches de Brasília, Distrito Federal. Rev. Bras. Saude M ater. Infant. 2005; 5(4):419-428.

24. Holland CV. A creche e seu papel na formação de práticas alimentares [dissertação]. São Paulo (SP): Faculdade de Saúde Pública, Universidade de São Paulo; 1999

25. Ferreira FCM, Osório M M. Consumo alimentar de macro e micronutrientes de crianças menores de cinco anos no Estado de Pernambuco, Brasil. Rev. Bras. Saude M ater. Infant. 2007; 7(1):63-74.

26. Pereira RC, Diniz AS, Ferreira LOC. N ew findings on iron absorption conditioning factors. Rev. Bras. Saude M ater. Infant. 2004; 4(3):241-248.

27. Oliveira MAA, Osório MM, Raposo MC F. Fatores socioeconômicos e dietéticos de risco para a anemia em crianças de 6 a 59 meses de idade. J. Pediatr. 2007; 83(1): 39-46.

Artigo apresentado em 20/11/2007

Aprovado em 28/07/2008

Versão final apresentada em 13/09/2008 